\title{
GROTHENDIECK ORDERED BANACH SPACES WITH AN INTERPOLATION PROPERTY
}

\author{
IOANNIS A. POLYRAKIS AND FOIVOS XANTHOS \\ (Communicated by Thomas Schlumprecht)
}

\begin{abstract}
In this paper we prove that if $E$ is an ordered Banach space with the countable interpolation property, $E$ has an order unit and $E_{+}$is closed and normal, then $E$ is a Grothendieck space; i.e. any weak-star convergent sequence of $E^{*}$ is weakly convergent. By the countable interpolation property we mean that for any $A, B \subseteq E$ countable, with $A \leq B$, we have $A \leq\{x\} \leq B$ for some $x \in E$.
\end{abstract}

\section{INTRODUCTION AND NOTATION}

A. Grothendieck proved in [8] that if $E=C(K)$, where $K$ is a compact and Hausdorff Stonian space, any weak-star convergent sequence of $E^{*}$ is weakly convergent; therefore, according to the current terminology, $E$ is a Grothendieck space. By the Kakutani Representation Theorem the above result can be formulated as follows: Any Dedekind complete AM-space with an order unit is a Grothendieck space.

G. Seever proved in [15] (see Theorem B and Theorem 1.1) that if $K$ is a compact and Hausdorff F-space, then $E=C(K)$ is a Grothendieck space or equivalently that any AM-space $E$ with the countable interpolation property and an order unit is a Grothendieck space. The countable interpolation property (see below) has been defined in [15], where it is referred to as property (I). So Seever improved the result of Grothendieck by replacing the Dedekind completeness of the space by the weaker one of the countable interpolation property.

P. G. Dodds studied in [7] the sequential convergence in the order dual $E^{\sim}$ of a Riesz space $E$ in the case where $E$ has the countable interpolation property. Specifically in Theorem 4.5, it is proved that if a sequence of $E^{\sim}$ is $\sigma\left(E^{\sim}, E\right)$ convergent, then it is convergent in the $\sigma\left(E^{\sim}, \operatorname{Id}(E)\right)$ topology of $E^{\sim}$, where $\operatorname{Id}(E)$ is the ideal generated by $E$ in the second order dual $\left(E^{\sim}\right)^{\sim}$ of $E$.

H. P. Lotz, in an old article of 1986 which has appeared recently, replaced the existence of an order unit in the result of Seever by a number of weaker conditions, [12], Theorem 1.

In this article, Theorem 9, we show that the result of Seever is true without the lattice condition. Specifically we show that if $E$ is an ordered Banach space with

Received by the editors February 14, 2011 and, in revised form, September 5, 2011.

2010 Mathematics Subject Classification. Primary 46B40, 46B42, 47 B60.

Key words and phrases. Ordered Banach spaces, Banach lattices, Grothendieck spaces, regular operators.

This research was supported by the HERAKLEITOS II project, which is co-funded by the European Social Fund and National Resources. 
the countable interpolation property, $E$ has an order unit and $E_{+}$is closed and normal, then $E$ is a Grothendieck space.

As an application of our theorem, in Corollary 11, we show that if the space of regular operators $\mathcal{L}^{r}(E, F)$, where $E, F$ are Banach lattices, has the countable interpolation property, then any order convex subspace $I_{T}$ of $E$ generated by a positive operator $T \in \mathcal{L}^{r}(E, F)$, equipped with the order unit norm $\|\cdot\|_{T}$, is a Grothendieck space.

In 17, Theorem 3.1, A. Wickstead proved that the space of regular operators $\mathcal{L}^{r}(c, F)$, where $c$ is the space of convergent real sequences and $F$ is a Banach lattice, has the countable interpolation property if and only if $F$ has the monotone countable interpolation property. Based on this result and on the Example 3.2 of the same article we give an example of an ordered Banach space that satisfies the conditions of our theorem, but it isn't a vector lattice.

In [6], N. Danet proved that if $E$ is a separable Banach lattice and $F$ is a Banach lattice with the countable interpolation property, then $\mathcal{L}^{r}(E, F)$ has the interpolation property. In this article (for the exact details, see Theorem 13) we prove that $\mathcal{L}^{r}(E, F)$ has the countable interpolation property in the case where $E, F$ are ordered Banach spaces, $E$ has a positive basis and $F$ has the countable interpolation property.

We present below different notions of order interpolation. In this article we use the term monotone countable interpolation property and countable interpolation property instead of the different ones which have been used in the literature because they express better the underlying properties. Let $E$ be a (partially) ordered vector space with positive cone $E_{+}$. Note that a convex subset $P \neq \emptyset$ of a vector space is a cone if $\lambda P=P$ for any real number $\lambda>0$ and $P \cap(-P)=\{0\} . E$ has the (finite) interpolation property if for all finite subsets $A, B$ of $E$ with $A \leq B$, (i.e. $a \leq b$ for each $a \in A, b \in B$ ) there exists $x \in E$ such that $A \leq\{x\} \leq B$. We say that $E$ has the Cantor property or the monotone countable interpolation property if for any increasing sequence $\left\{x_{n}\right\}$ and any decreasing sequence $\left\{y_{n}\right\}$ of $E$ with $x_{n} \leq y_{n}$ for each $n$, there exists $x \in E$ such that $x_{n} \leq x \leq y_{n}$ for each $n$. $E$ has the countable interpolation property if for any $\left\{x_{n}\right\},\left\{y_{n}\right\}$ sequences of $E$ with $x_{n} \leq y_{m}$ for each $n, m$, there exists $x \in E$ such that $x_{n} \leq x \leq y_{n}$ for each $n$. If $E$ is a vector lattice (Riesz space), the monotone countable interpolation property and the countable interpolation property are equivalent, but in general these notions are not equivalent. Vector lattices do not always have the countable interpolation property. Indeed by [15] we have that $C(K)$, where $K$ is a compact and Hausdorff topological space, has the monotone countable interpolation property if and only if $K$ is an F-space. C. Huijsmans and B. de Pagter proved in [9], Theorem 9.15, that if $E$ is an Archimedean vector lattice, then $E$ has the monotone countable interpolation property if and only if $E$ is uniformly complete and $\left\{x^{+}\right\}^{d}+\left\{x^{-}\right\}^{d}=E$, for any $x \in E$. If $E_{+}$is generating and $E$ has the interpolation property, the RieszKantorovich formula is valid for the vectors of the order dual $E^{\sim}$ of $E$. Recall that $E^{\sim}$ is the set of ordered bounded linear functionals of $E$ and that the interpolation property and the Riesz decomposition property are equivalent.

If $E$ is a vector lattice, the solid hull, $\operatorname{Sol}(A)$, of a subset $A$ of $E$ is the smallest solid set that contains $A$. A subset $B$ of $E$ is solid if $x \in B,|y| \leq|x|$ implies $y \in B$.

Suppose that $E$ is an ordered normed space. Denote by $E^{*}$ the topological dual of $E$. If a real number $c>0$ exists so that $0 \leq x \leq y$ implies $\|x\| \leq c\|y\|$, then 
the cone $E_{+}$is normal and $c$ is a constant of the normal cone $E_{+}$. If $e$ is an order unit of $E$, the norm $\|x\|_{e}=\inf \{\lambda>0 \mid x \in[-\lambda e, \lambda e]\}$ is the order unit norm of $E$ induced by $e$. If $E$ has the interpolation property and $E_{+}$is closed, generating and normal, then $E^{*}$ is a Banach lattice with respect to an equivalent norm; see for example [4, Theorem 2.47, and the comments in Exercise 17, page 98. A Banach space $X$ is a Grothendieck space if any weak-star convergent sequence of $X^{*}$ is weakly convergent. Trivial examples of Grothendieck spaces are the reflexive spaces and of non-Grothendieck the non-reflexive, separable spaces. For some recent results on Grothendieck spaces, independent from this article, we refer to [10, [2] and 14]. In [14, Theorem 15, the following cone characterization is proved, which unfortunately cannot be applied, at least directly in this article. A Banach space $X$ is non-Grothendieck if and only if there exists a well-based cone $P$ of $X^{*}$ such that $\operatorname{int}\left(P_{0}\right)=\emptyset$ and the set of quasi-interior points of $P_{0}$ with respect to the seminorm $d_{P}(x)=\sup \left\{\left|x^{*}(x)\right| \quad \mid x^{*} \in P,\left\|x^{*}\right\| \leq 1\right\}$ of $X$ is nonempty, where $P_{0}=\left\{x \in X \mid x^{*}(x) \geq 0\right.$, for any $\left.x^{*} \in P\right\}$ is the dual cone of $P$ in $X$.

Note that the cone $P$ is well-based if a strictly positive and continuous linear functional $f$ of $X^{*}$ exists so that the set $B=\left\{x^{*} \in P \mid f\left(x^{*}\right)=1\right\}$ is bounded. Note also in the above theorem that $X$ is ordered by the dual cone $P_{0}$ of $P$ in $X$ and also that $x_{0} \in P_{0}$ is a quasi-interior point of $P_{0}$ with respect to the seminorm $d_{P}$ if the subspace $\bigcup_{n=1}^{\infty}\left[-n x_{0}, n x_{0}\right]$ is $d_{P}$-dense in $X$. Of course, if $x_{0}$ is a quasiinterior point with respect to the norm of $X$, then it is also a quasi-interior point with respect to the seminorm $d_{P}$.

\section{THE RESUlts}

In this section we will denote by $E$ an ordered vector space. The notion of an $l^{1}$-sequence of $E$ and the notion of the equi- $l^{1}$-continuous subset of $E^{\sim}$ have been defined by $\mathrm{O}$. Burkinshaw in [5], where the weakly compact sets in the order dual $E^{\sim}$ of $E$ are studied, in the case where $E$ is a vector lattice. In this article we use these definitions in ordered vector spaces.

Definition 1. A sequence $\left\{x_{n}\right\} \subseteq E$ is an $l^{1}$-sequence if there exists $x \in E_{+}$and a sequence $\left\{b_{n}\right\} \subseteq E_{+}$such that $-b_{n} \leq x_{n} \leq b_{n}$ and $\sum_{i=1}^{n} b_{i} \leq x$ for each $n$.

For any $\sigma\left(E^{\sim}, E\right)$-bounded subset $A$ of $E^{\sim}$, denote by $\rho_{A}$ the following seminorm on $E$ :

$$
\rho_{A}(x)=\sup \{|y(x)| \mid y \in A\}, \text { for each } x \in E .
$$

Definition 2. A subset $A$ of $E^{\sim}$ is equi- $l^{1}$-continuous if $A$ is $\sigma\left(E^{\sim}, E\right)$-bounded and $\lim _{n \rightarrow \infty} \rho_{A}\left(x_{n}\right)=0$ for any $l^{1}$-sequence $\left\{x_{n}\right\}$ of $E$.

Proposition 3. Suppose that $A$ is a $\sigma\left(E^{\sim}, E\right)$-bounded subset of $E^{\sim}$. If $\lim _{n \rightarrow \infty} \rho_{A}\left(x_{n}\right)$ $=0$ for each positive $l^{1}$-sequence $\left\{x_{n}\right\}$ of $E$, then $A$ is equi-l ${ }^{1}$-continuous.

Proof. Suppose that $\left\{x_{n}\right\}$ is an $l^{1}$-sequence of $E$. Then there exists $\left\{b_{n}\right\} \subseteq E_{+}$, $x \in E_{+}$such that $-b_{n} \leq x_{n} \leq b_{n}$ and $\sum_{i=1}^{n} b_{i} \leq x$. Note that $\left\{b_{n}\right\}$ is a positive $l^{1}$-sequence. Also $\left\{x_{n}+b_{n}\right\}$ is a positive $l^{1}$-sequence, because $\sum_{i=1}^{n}\left(x_{i}+b_{i}\right)$ $\leq \sum_{i=1}^{n} b_{i}+x \leq 2 x$, for each $n$. Therefore $\rho_{A}\left(x_{n}\right)=\rho_{A}\left(\left(x_{n}+b_{n}\right)-b_{n}\right) \leq$ $\rho_{A}\left(x_{n}+b_{n}\right)+\rho_{A}\left(b_{n}\right)$, and from our hypothesis we have that $\lim _{n \rightarrow \infty} \rho_{A}\left(x_{n}\right)=0$. 
Proposition 4. Suppose that $E_{+}$is generating and $E$ has the interpolation property. If $A$ is a subset of $E^{\sim}$ and the solid hull, Sol $(A)$, of $A$ in $E^{\sim}$ is $\sigma\left(E^{\sim}, E\right)$ bounded, we have: The set $A$ is equi-l $l^{1}$-continuous if and only if $\operatorname{Sol}(A)$ is equi-l ${ }^{1}$ continuous.

Proof. If the set $B=\operatorname{Sol}(A)$ is equi- $l^{1}$-continuous, $A$ is equi- $l^{1}$-continuous. Suppose that $A$ is equi $-l^{1}$ continuous. If $B$ is not equi- $l^{1}$-continuous, by Proposition 3 , there exists $\epsilon>0$ and a positive $l^{1}$-sequence $\left\{x_{n}\right\}$, such that $\rho_{B}\left(x_{n}\right)>\epsilon$ for each $n \in \mathbb{N}$, so there exists a sequence $\left\{z_{n}\right\} \subseteq B$ such that $\left|z_{n}\left(x_{n}\right)\right|>\epsilon$ for each $n$. Since $B=\operatorname{Sol}(A)$ there exists a sequence $\left\{y_{n}\right\}$ of $A$ such that $\left|z_{n}\right| \leq\left|y_{n}\right|$; therefore

$$
\left|y_{n}\right|\left(x_{n}\right) \geq\left|z_{n}\right|\left(x_{n}\right) \geq\left|z_{n}\left(x_{n}\right)\right|>\epsilon,
$$

and by the Riesz-Kantorovich formula we have

$$
\left|y_{n}\right|\left(x_{n}\right)=\sup \left\{y_{n}(u) \mid-x_{n} \leq u \leq x_{n}\right\} .
$$

So there exists a sequence $\left\{u_{n}\right\}$ of $E$ such that $-x_{n} \leq u_{n} \leq x_{n}$ and $y_{n}\left(u_{n}\right) \geq \epsilon$, for each $n$. This is a contradiction because $\left\{u_{n}\right\}$ is an $l^{1}$-sequence and $A$ is equi- $l^{1}$ continuous.

Proposition 5. If $A \subseteq E^{\sim}$, and $A$ is $\sigma\left(E^{\sim}, E\right)$-bounded the following are equivalent:

(i) A is equi-l ${ }^{1}$-continuous.

(ii) Every order bounded increasing sequence of $E$ is $\rho_{A}$-Cauchy.

Proof. $(i) \Rightarrow(i i)$ : Suppose that $(i i)$ is not true. Then there exists an order bounded increasing sequence $\left\{x_{m}\right\}$ of $E$ which is not $\rho_{A}$-Cauchy. So there exists $\epsilon>0$ and a strictly increasing sequence $\left\{m_{n}\right\}$ of $\mathbb{N}$ such that

$$
\rho_{A}\left(x_{m_{n+1}}-x_{m_{n}}\right)>\epsilon \text { for each } n \in \mathbb{N} .
$$

If $\left\{x_{m}\right\}$ is dominated by $x$, the sequence $y_{n}=x_{m_{n+1}}-x_{m_{n}}$ is an $l^{1}$-sequence because $0 \leq \sum_{i=1}^{n} y_{i}=x_{m_{n+1}}-x_{m_{1}} \leq x-x_{m_{1}}$, for each $n$. By (11) we have $\rho_{A}\left(y_{n}\right)>\epsilon$ for each $n$, which contradicts $(i)$.

$($ ii $) \Rightarrow(i)$ : Suppose that $\left\{x_{n}\right\}$ is a positive $l^{1}$-sequence. Then the sequence $y_{n}=\sum_{i=1}^{n} x_{i}$ is order bounded and increasing; therefore by $(i i)$, it is $\rho_{A}$-Cauchy. So we have $\lim \rho_{A}\left(x_{n}\right)=\lim \rho_{A}\left(y_{n}-y_{n-1}\right)=0$. Therefore $A$ is equi- $l^{1}$-continuous, by Proposition 3

The topological dual $\ell_{\infty}^{*}$ of $\ell_{\infty}$ is the direct sum

$$
\ell_{\infty}^{*}=\ell_{1} \oplus \ell_{1}^{d},
$$

where $\ell_{1}^{d}$ is the disjoint complement of $\ell_{1}$ in $\ell_{\infty}^{*}$. So each $x \in \ell_{\infty}^{*}$ is the sum $x=x^{1}+x^{2}$, where $x^{1}$ and $x^{2}$ are the components of $x$ in $\ell_{1}$ and $\ell_{1}^{d}$. By the Phillips Lemma (see for example [3], Theorem 4.67), for any sequence $\left\{x_{n}\right\}$ of $\ell_{\infty}^{*}$ such that $x_{n} \stackrel{w^{*}}{\longrightarrow} 0$, we have $x_{n}^{1} \stackrel{\|\cdot\|}{\longrightarrow} 0$; i.e., the sequence of the $\ell_{1}$-components of the sequence $\left\{x_{n}\right\}$ converges to zero. Recall also that by Lozanovsky's theorem (see for example [4, Theorem 2.32), if X, $Y$ are ordered Banach spaces with closed positive cones and $X_{+}$is generating, then every linear, positive operator $T: X \rightarrow Y$ is continuous.

Theorem 6. Let $E$ be an ordered Banach space with closed, normal and generating positive cone $E_{+}$. If $E$ has the countable interpolation property and $\left\{x_{n}^{*}\right\}$ is a sequence of $E^{*}$ so that $x_{n}^{*} \stackrel{w^{*}}{\longrightarrow} 0$, then the set $A=\left\{x_{n}^{*}\right\}$ is equi-l $l^{1}$-continuous. 
Proof. Note that $E^{\sim}=E^{*}$ and also that $A$ is bounded. Suppose that $A=\left\{x_{n}^{*}\right\}$ is not equi- $l^{1}$-continuous. Then by Proposition 3, there exists a positive $l^{1}$-sequence $\left\{x_{n}\right\}$ of $E$ with $\rho_{A}\left(x_{n}\right)>\epsilon$, for each $n$. Since $\left\{x_{n}\right\}$ is a positive $l^{1}$-sequence, there exists $x \in E_{+}$so that

$$
0 \leq \sum_{i=1}^{n} x_{i} \leq x, \text { for each } n .
$$

Also by the relation $\rho_{A}\left(x_{n}\right)>\epsilon$, for each $n$ there exists a real number $k_{n}$, so that $\left|x_{k_{n}}^{*}\left(x_{n}\right)\right|>\epsilon$. We assert that the set $K=\left\{k_{n} \mid n \in \mathbb{N}\right\}$ is infinite because if we suppose that this set is finite we have a contradiction as follows: $0 \leq\left|x_{k_{n}}^{*}\right|\left(\sum_{i=1}^{n} x_{i}\right) \leq\left|x_{k_{n}}^{*}\right|(x)$; therefore $\lim _{i \longrightarrow \infty}\left|x_{k_{n}}^{*}\right|\left(x_{i}\right)=0$, for each $k_{n}$. Hence there exists $i_{0} \in \mathbb{N}$ so that $\left|x_{k_{n}}^{*}\left(x_{i}\right)\right| \leq\left|x_{k_{n}}^{*}\right|\left(x_{i}\right)<\epsilon$ for each $i \geq i_{0}$ and each $k_{n} \in K$.

So the set $K$ is infinite. Therefore there exists a subsequence of $\left\{x_{n}^{*}\right\}$ which we denote again with $\left\{x_{n}^{*}\right\}$ such that

$$
\left|x_{n}^{*}\left(x_{n}\right)\right|>\epsilon, \text { for each } n .
$$

Let

$$
\phi=\sum_{i=1}^{\infty} \frac{\left|x_{i}^{*}\right|}{2^{i}},
$$

$I_{\phi}=\bigcup_{n=1}^{\infty}[-n \phi, n \phi]$ be the ideal of $E^{*}$ generated by $\phi$ and $M=\left\{x \in E \mid x^{*}(x)=\right.$ 0 , for any $\left.x^{*} \in I_{\phi}\right\}$ be the annihilator of $I_{\phi}$ in $E$.

We define below a positive operator $T: \ell_{\infty} \rightarrow E / M$. For each $a=\left(a_{i}\right) \in \ell_{\infty}^{+}$we put $f_{n}^{a}=\sum_{i=1}^{n} a_{i} x_{i}$, for each $n \in \mathbb{N}$. By (2),$f_{n}^{a} \leq\left\|\left(a_{i}\right)\right\| x$; hence the set of upper bounds

$$
U_{a}=\left\{w \in E \mid f_{n}^{a} \leq w, \text { for each } n\right\}
$$

of $\left\{f_{n}^{a}\right\}$, is nonempty. We show below that $\phi$ restricted on $U_{a}$ takes a minimum on a subset $S_{a}$ of $U_{a}$ and that for each $A \subseteq U_{a}$ finite there exists $v \in S_{a}$ with $A \geq\{v\}$. Indeed, if $\left\{g_{n}\right\} \subseteq U_{a}$ such that

$$
\inf \left\{\phi\left(g_{n}\right) \mid n \in \mathbb{N}\right\}=\inf \left\{\phi(w) \mid w \in U_{a}\right\},
$$

by the countable interpolation property there exists $u \in E$ with $f_{n}^{a} \leq u \leq g_{n}$ for each $n$; therefore $u \in S_{a}$. Also for any $A \subseteq U_{a}$ we have $A \cup\{u\} \geq\left\{f_{n}^{a}\right\}$, where $u \in S_{a}$. Hence there exists $v \in E$ such that $A \cup\{u\} \geq\{v\} \geq\left\{f_{n}^{a}\right\}$; therefore $v \in S_{a}$.

Let $\pi: E \rightarrow E / M$ with $\pi(x)=x+M$ be the quotient map. For any $a=\left(a_{i}\right) \in$ $\ell_{\infty}^{+}$we put

$$
T(a)=\pi(u), \text { where } u \in S_{a} .
$$

We will show that $\pi(u)=\pi(v)$ for any $u, v \in S_{a}$. Therefore $T$ is well defined. First we note that $\operatorname{Ker}(\phi) \cap E_{+}=M \cap E_{+}$and $S_{a} \subseteq E_{+}$. Since $u, v \in U_{a}$ there exists $w \in S_{a}$ with $u, v \geq w$. So $u-w, v-w \in \operatorname{Ker}(\phi) \cap E_{+} \subseteq M$; therefore $u-v=(u-w)-(v-w) \in M$ and $T$ is well defined. We will show that $T$ is positive homogeneous and additive, so we suppose that

$$
a=\left(a_{i}\right), b=\left(b_{i}\right) \in \ell_{\infty}^{+}, T(a)=\pi(u), T(b)=\pi(v) \text { and } T((a+b))=\pi(z) .
$$

It is easy to show that $U_{\lambda a}=\lambda U_{a}$ for any $\lambda>0$; therefore $T$ is positive homogeneous. Since $U_{a}+U_{b} \subseteq U_{a+b}$, we have $u+v \in U_{a+b}$; therefore $\phi(u+v) \geq \phi(z)$. Also $z \geq f_{n}^{a}+f_{m}^{b}$ for each $n, m$ because if $n \geq m, z \geq f_{n}^{a+b}=f_{n}^{a}+f_{n}^{b} \geq f_{n}^{a}+f_{m}^{b}$. Therefore there exists $h \in E$ such that $z-f_{n}^{a} \geq h \geq f_{m}^{b}$ for each $n, m$. So $h \in U_{b}$; therefore 
there exists $w \in S_{b}$ such that $z-f_{n}^{a} \geq h \geq w$ for each $n$. So we have $z-w \geq f_{n}^{a}$ for each $n$. Therefore $z-w \in U_{a}$; hence there exists $p \in S_{a}$ such that $z-w \geq p$. So we have $\phi(z) \geq \phi(w)+\phi(p)=\phi(u+v)$. Therefore $T(a+b)=T(a)+T(b)$.

For any $a \in \ell_{\infty}$ we put $T(a)=T\left(a^{+}\right)-T\left(a^{-}\right) . \pi\left(E_{+}\right)$is closed because the quotient map is open. Also $\pi\left(E_{+}\right)$is a cone because if we suppose that $\pm w \in \pi\left(E_{+}\right)$ we have $w=\pi(x),-w=\pi(y)$, where $x, y \in E_{+}$; therefore $\pi(x+y)=\pi(0)$. So we have $x+y \in M \cap E_{+}$; therefore $\phi(x+y)=0$, which implies that $\phi(x)=\phi(y)=0$ because $x, y \in E_{+}$. Hence $x, y \in \operatorname{Ker}(\phi) \cap E_{+}=M \cap E_{+}$and $w=\pi(x)=0$. By the Lozanovsky Theorem, $T$ is continuous. Also for any $n$ we have $T\left(e_{n}\right)=\pi\left(x_{n}\right)$, where $e_{n}$ is the vector of $\ell_{\infty}$ with the value one in the $n$-position and zero elsewhere.

The spaces $(E / M)^{*}$ and $M^{\perp}=\left\{x^{*} \in E^{*} \mid x^{*}(x)=0\right.$, for each $\left.x \in M\right\}$ are isometric with $x^{*}(\pi(x))=x^{*}(x)$ for each $x^{*} \in M^{\perp}$. Note that $x_{n}^{*} \in M^{\perp}$ because $\frac{\left|x_{n}^{*}\right|}{2^{n}} \leq \phi$ for each $n$ and $I_{\phi} \subseteq M^{\perp}$. Also $T^{*}\left(x_{n}^{*}\right) \stackrel{w^{*}}{\longrightarrow} 0$ because $T^{*}$ is $w^{*}$ to $w^{*}$ continuous. By the Phillips Lemma (and the notation before the theorem) we have

$$
\left(T^{*}\left(x_{n}^{*}\right)\right)^{1} \stackrel{\|\cdot\|}{\longrightarrow} 0
$$

therefore

$$
x_{n}^{*}\left(x_{n}\right)=T^{*}\left(x_{n}^{*}\right)\left(e_{n}\right)=\left(T^{*}\left(x_{n}^{*}\right)\right)^{1}\left(e_{n}\right)+\left(T^{*}\left(x_{n}^{*}\right)\right)^{2}\left(e_{n}\right)=\left(T^{*}\left(x_{n}^{*}\right)\right)^{1}\left(e_{n}\right),
$$

because $\left(T^{*}\left(x_{n}^{*}\right)\right)^{2}\left(e_{n}\right)=0$ for each $n$. By (3) we have that $\left|\left(T^{*}\left(x_{n}^{*}\right)\right)^{1}\left(e_{n}\right)\right|>$ $\epsilon$, which contradicts (4) and the theorem is true.

Lemma 7. Suppose that $E$ is an ordered vector space with the countable interpolation property and $\left\{x_{n}\right\}$ is a sequence of $E_{+}$.

(i) If the sequence $\left\{x_{n}\right\}$ is dominated by $x \in E$, there exists an increasing sequence $\left\{u_{n}\right\}$ of $E_{+}$, such that

$$
x_{1}, \ldots, x_{n} \leq u_{n} \leq \sum_{i=1}^{n} x_{i}, x, \text { for each } n .
$$

(ii) If $\left\{y_{n}\right\}$ is a sequence of $E$ such that $\left\{x_{1}, \ldots, x_{n}\right\} \geq\left\{y_{m} \mid m \geq n\right\}$ for each $n$, there exists a decreasing sequence $\left\{w_{n}\right\}$ of $E_{+}$, such that

$$
\left\{x_{1}, \ldots, x_{n}\right\} \geq\left\{w_{n}\right\} \geq\left\{y_{m} \mid m \geq n\right\}, \text { for each } n .
$$

Proof. (i) We put $u_{1}=x_{1}$. Then $x_{1}, x_{2} \leq x_{1}+x_{2}, x$ and by the interpolation property, there exists $u_{2} \in E$ so that $x_{1}, x_{2} \leq u_{2} \leq x_{1}+x_{2}, x$. We have $x_{1}, x_{2}, x_{3}, u_{2} \leq$ $x_{1}+x_{2}+x_{3}, x$ so there exists $u_{3} \in E$ so that $x_{1}, x_{2}, x_{3}, u_{2} \leq u_{3} \leq x_{1}+x_{2}+x_{3}, x$, and continuing this process we have that (5) is true for each $n$.

(ii) $\{0\} \cup\left\{y_{m} \mid m \geq 1\right\} \leq x_{1}$, so there exists $w_{1}$ with $\{0\} \cup\left\{y_{m} \mid m \geq 1\right\} \leq$ $\left\{w_{1}\right\} \leq\left\{x_{1}\right\}$. Therefore $\{0\} \cup\left\{y_{m} \mid m \geq 2\right\} \leq\left\{w_{1}\right\} \cup\left\{x_{1}, x_{2}\right\}$ and by the countable interpolation property, there exists $w_{2} \in E$ so that $\{0\} \cup\left\{y_{m} \mid m \geq 2\right\} \leq\left\{w_{2}\right\} \leq$ $\left\{w_{1}\right\} \cup\left\{x_{1}, x_{2}\right\}$, and continuing we have that (6) is true for each $n$.

Theorem 8. Let $E$ be an ordered Banach space with closed, normal and generating positive cone $E_{+}$. If $E$ has the countable interpolation property, then for any equi$l^{1}$-continuous $A \subseteq E^{*}$, for any $x \in E_{+}$and for any $\epsilon>0$, there exists $y^{*} \in E_{+}^{*}$ such that

$$
\left(\left|x^{*}\right|-y^{*}\right)^{+}(x)<\epsilon,
$$

for each $x^{*} \in A$. 
Proof. It is enough to show the theorem with the extra assumption that $A$ is solid because by Proposition 4 $A$ is equi- $l^{1}$-continuous if and only if $\operatorname{Sol}(A)$ is equi- $l^{1}$ continuous. So we suppose that $A$ is solid. Suppose that the theorem is not true. Then there exists $\epsilon>0$ and $x \in E_{+}$such that for each $y^{*} \in E_{+}^{*}$ there exists $x^{*} \in A$ such that

$$
\left(\left|x^{*}\right|-y^{*}\right)^{+}(x)>2 \epsilon .
$$

As we show below, a sequence $\left\{x_{n}^{*}\right\}$ of $A_{+}=A \cap E_{+}^{*}$ exists so that

$$
\left(x_{n+1}^{*}-2^{n-1} \sum_{i=1}^{n} x_{i}^{*}\right)^{+}(x)>2 \epsilon, \text { for each } n .
$$

Indeed, if we suppose in (7) that $y^{*} \in A_{+}$and we put $x_{1}^{*}=y^{*}$ and $x_{2}^{*}=\left|x^{*}\right|$, then $x_{2}^{*} \in A_{+}$because $x^{*} \in A$ and $A$ is solid and the relation is true for $n=1$. If in (77) we put $y^{*}=2\left(x_{1}^{*}+x_{2}^{*}\right)$ and $x_{3}^{*}=\left|x^{*}\right|$, we have that $x_{3}^{*} \in A_{+}$. Therefore (8) is true for $n=2$, and continuing this process we have that (8) is true for each $n$. By the Riesz-Kantorovich formula, $\left(z^{*}\right)^{+}(x)=\sup \left\{z^{*}(y) \mid y \in[0, x]\right\}$ for any $z^{*} \in E^{*}$. Therefore by (8), a sequence $\left\{y_{n}\right\}$ of $[0, x]$ exists such that

$$
\left(x_{n+1}^{*}-2^{n-1} \sum_{i=1}^{n} x_{i}^{*}\right)\left(y_{n}\right)>2 \epsilon, \text { for each } n .
$$

For each $n \in \mathbb{N},\left\{y_{n+k} \mid k \in \mathbb{N}\right\}$ is a sequence of the interval $[0, x]$ of $E$. Therefore by Lemma 7, an increasing sequence $\left\{u_{n k} \mid k \in \mathbb{N}\right\}$ of $E_{+}$exists so that

$$
y_{n}, y_{n+1}, \ldots, y_{n+k} \leq u_{n k} \leq \sum_{i=n}^{n+k} y_{i}, x, \text { for each } k .
$$

Since $\left\{u_{n k}\right\}$ is an increasing sequence of the interval $[0, x]$, by Proposition 5 , it is $\rho_{A}$-Cauchy. Therefore a natural number $k_{n}$ exists so that

$$
\rho_{A}\left(u_{n k}-u_{n k_{n}}\right)<2^{-n} \epsilon \text { for each } k \geq k_{n} .
$$

Suppose that $m \geq n$. Then for each $k \geq m, k_{n}$ we have $y_{m} \leq u_{n k}$. Therefore

$$
y_{m}-u_{n k_{n}} \leq u_{n k}-u_{n k_{n}} \text { and } u_{n k}-u_{n k_{n}} \geq 0 .
$$

So there exists $z_{n m} \in E_{+}$so that

$$
\left\{0, y_{m}-u_{n k_{n}}\right\} \leq\left\{z_{n m}\right\} \leq\left\{u_{n k}-u_{n k_{n}} \mid k \geq m, k_{n}\right\}
$$

So we have defined a double sequence $\left\{z_{n m} \mid n \in \mathbb{N}, m \geq n\right\}$ of $E_{+}$for which we have

$$
x_{n+1}^{*}\left(z_{i n}\right) \leq \rho_{A}\left(u_{i k}-u_{i k_{i}}\right) \leq 2^{-i} \epsilon,
$$

for each $i \leq n$ and each $k \geq n, k_{i}$. By (12), for any $j=1,2, \ldots, n$ and $m \geq n$, we have

$$
u_{j k_{j}} \geq y_{m}-z_{j m} ; \text { therefore } u_{j k_{j}} \geq y_{m}-\sum_{i=1}^{m} z_{i m} .
$$

By (ii) of Lemma 7, a decreasing sequence $\left\{w_{n}\right\}$ of $E_{+}$exists so that

$$
\left\{y_{m}-\sum_{i=1}^{m} z_{i m} \mid m \geq n\right\} \leq\left\{w_{n}\right\} \leq\left\{u_{1 k_{1}}, u_{2 k_{2}}, \ldots, u_{n k_{n}}\right\}, \text { for each } n \in \mathbb{N} .
$$


Then $\left\{w_{1}-w_{n}\right\}$ is an increasing sequence dominated by $w_{1}$ and according to Proposition 5 , $\left\{w_{1}-w_{n}\right\}$, is $\rho_{A}$-Cauchy. This is a contradiction because as we will show below, $\left\{w_{1}-w_{n}\right\}$ is not $\rho_{A}$-Cauchy; therefore the theorem is true.

We prove this assertion as follows: For each $n$ we have $w_{n} \geq y_{n}-\sum_{i=1}^{n} z_{i n}$. Therefore

$$
x_{n+1}^{*}\left(w_{n}\right) \geq x_{n+1}^{*}\left(y_{n}\right)-x_{n+1}^{*}\left(\sum_{i=1}^{n} z_{i n}\right) .
$$

By (9) we have

$$
x_{n+1}^{*}\left(y_{n}\right)>2 \epsilon
$$

and by (13),$\quad \sum_{i=1}^{n} x_{n+1}^{*}\left(z_{i n}\right) \leq \epsilon \sum_{i=1}^{n} 2^{-i}$. Therefore we have

$$
x_{n+1}^{*}\left(w_{n}\right) \geq 2 \epsilon-\epsilon \sum_{i=1}^{n} 2^{-i}>\epsilon, \text { for each } n .
$$

Also, for any $w_{r}$ we have $x_{n+1}^{*}\left(w_{r}\right) \leq x_{n+1}^{*}\left(u_{r k_{r}}\right)$ and by (10) we have $u_{r k_{r}} \leq$ $\sum_{i=r}^{r+k_{r}} y_{i}$. Therefore

$$
x_{n+1}^{*}\left(w_{r}\right) \leq x_{n+1}^{*}\left(\sum_{i=r}^{r+k_{r}} y_{i}\right) \leq \sum_{i=r}^{\infty} x_{n+1}^{*}\left(y_{i}\right) .
$$

By (9) we have $x_{i}^{*}\left(y_{n}\right) \leq 2^{-n+1} x_{n+1}^{*}\left(y_{n}\right)$ for each $n \geq i$. Therefore, if $M$ is a norm bound of $A$ and $c$ a constant of the normal cone $E_{+}$, we have

$$
x_{i}^{*}\left(y_{n}\right) \leq M c\|x\| 2^{-n+1}, \text { for each } n \geq i,
$$

because $\left\{y_{n}\right\}$ is a sequence of $[0, x]$. Therefore

$$
x_{n+1}^{*}\left(w_{n+1}\right) \leq \sum_{i=n+1}^{\infty} x_{n+1}^{*}\left(y_{i}\right) \leq \sum_{i=n+1}^{\infty} 2^{-i+1} M\|x\| c=2^{-n+1} M c\|x\| \text { for each } n \text {. }
$$

So, by the definition of $\rho_{A}$ and by (16) and (17) we have

$$
\rho_{A}\left(w_{n}-w_{n+1}\right) \geq x_{n+1}^{*}\left(w_{n}-w_{n+1}\right) \geq \epsilon-2^{-n+1} M\|x\| c,
$$

for each $n$. Therefore $\left\{w_{1}-w_{n}\right\}$ is not $\rho_{A}$-Cauchy and the theorem is true.

Theorem 9. Let $E$ be an ordered Banach space with closed and normal positive cone $E_{+}$. If $E$ has an order unit e and $E$ has the countable interpolation property, then $E$ is a Grothendieck space.

Proof. By 4, Theorem 2.63, the order unit norm and the initial norm of $E$ are equivalent, and suppose that $E$ is equipped with the order unit norm, which we denote by $\|$.$\| . Suppose that A=\left\{x_{n}^{*}\right\} \subseteq E^{*}$ and $x_{n}^{*} \stackrel{w^{*}}{\longrightarrow} 0$. By Theorem 6 , $A$ is equi- $l^{1}$-continuous. Therefore by Theorem 8 , for any $\epsilon>0$, there exists $y^{*} \in E_{+}^{*}$ so that

$$
\left\|\left(\left|x^{*}\right|-y^{*}\right)^{+}\right\|=\left(\left|x^{*}\right|-y^{*}\right)^{+}(e)<\epsilon, \text { for each } x^{*} \in A .
$$

Then we have

$$
\left|x^{*}\right|=\left|x^{*}\right| \wedge y^{*}+\left(\left|x^{*}\right|-y^{*}\right)^{+} \in\left[0, y^{*}\right]+\epsilon U_{E^{*}},
$$

where $U_{E^{*}}$ is the unit ball of $E^{*}$; therefore

$$
A \subseteq\left[-y^{*}, y^{*}\right]+\epsilon U_{E^{*}} .
$$


By the Dunford-Pettis Theorem, [13, Theorem 2.5.4, $A$ is a relative weakly compact subset of the AL-space $E^{*}$. If we suppose that $x_{n}^{*} \underset{\psi}{\rightarrow} 0$, there exists a subsequence $\left\{x_{k_{n}}^{*}\right\}$ of $\left\{x_{n}^{*}\right\}$ and $f \in E^{* *}$ such that $\inf \left\{f\left(x_{k_{n}}^{*}\right)\right\}>0$. This leads to a contradiction as follows: The set $\left\{x_{k_{n}}^{*}\right\}$ has a weakly convergent subsequence to a point $x_{0}^{*} \in E^{*}$. Since $x_{n}^{*} \stackrel{w^{*}}{\longrightarrow} 0$ we have that $x_{0}^{*}=0$, but this is impossible because $\inf \left\{f\left(x_{k_{n}}^{*}\right)\right\}>0$. Therefore $x_{n}^{*} \stackrel{w}{\longrightarrow} 0$ and $E$ is a Grothendieck space.

Let $K$ be a nonempty, compact and convex subset of a locally convex Hausdorff topological vector space. Then the space $A(K)$ of affine continuous real-valued functions on $K$ with the pointwise ordering and the supremum norm is an ordered Banach space and the constant function 1 is an order unit of $A(K)$, but $A(K)$ is not necessarily a lattice. By [11, Theorem 6, page 16 and 4, Theorem 2.63, any ordered Banach space $E$, with a closed and normal positive cone $E_{+}$and an order unit is order isomorphic with an $A(K)$ space, where $K$ is as above. Hence, under the above notation, Theorem 9 can be stated equivalently as follows:

Theorem 10. Any $A(K)$ space with the countable interpolation property is a Grothendieck space.

Recall that if $E, F$ are ordered normed spaces, denote by $\mathcal{L}(E, F)$ the space of bounded operators of $E$ into $F$ and by $\mathcal{L}^{r}(E, F)$ the space of regular operators, i.e. the space of bounded operators of $E$ into $F$ which are the difference of two positive operators. Also for any $e \in E_{+}$denote by $I_{e}=\bigcup_{n \in \mathbb{N}}[-n e, n e]$, the order convex subspace of $E$ generated by $e$. Then $\|x\|_{e}=\inf \{\lambda>0 \mid x \in[-\lambda e, \lambda e]\}$ is the order unit norm of $I_{e}$.

If $E, F$ are Banach lattices, then $\mathcal{L}^{r}(E, F)$, equipped with the regular operator norm $\|T\|_{r}=\inf \left\{\| S||\left|S \in \mathcal{L}_{+}(E, F),\right| T(x) \mid \leq S(|x|) \forall x \in E_{+}\right\}$, is a Banach space and its positive cone $\mathcal{L}_{+}(E, F)$ is generating, $\|\cdot\|_{r}$-closed and $\|\cdot\|_{r}$-normal.

Corollary 11. Suppose that E, F are Banach lattices. If the space of regular operators $\mathcal{L}^{r}(E, F)$ of $E$ into $F$ has the countable interpolation property, then for every $T \in \mathcal{L}_{+}(E, F)$, the order convex subspace $I_{T}$ of $\mathcal{L}^{r}(E, F)$ generated by $T$, equipped with the order unit norm $\|\cdot\|_{T}$, is a Grothendieck space.

Proof. First we note that $I_{T}$, as an order convex subspace of $\mathcal{L}^{r}(E, F)$, has the countable interpolation property. By [4], Theorem 2.55 and Theorem 2.60, the positive cone $I_{T}^{+}=I_{T} \cap \mathcal{L}_{+}(E, F)$ of $I_{T}$ is $\|\cdot\|_{T}$-closed and $\left(I_{T},\|\cdot\|_{T}\right)$ is a Banach space. Also $I_{T}^{+}$is $\|\cdot\|_{T}$-normal. Therefore by Theorem $9,\left(I_{T},\|\cdot\|_{T}\right)$ is a Grothendieck space.

We complete this study with an example of an order convex subspace $I_{T}$ generated by a positive vector $T$ of an $\mathcal{L}^{r}(E, F)$ space which (the space $I_{T}$ ) is not a vector lattice. Hence $I_{T}$ is an example of an ordered Banach space that satisfies the conditions of Theorem [9, but is not a vector lattice. In Example 3.2 of [17, it is noted that if $F=C(K)$, where $F$ has the countable interpolation property but $F$ is not $\sigma$-Dedekind complete, then, by [1], Theorem $3.10, \mathcal{L}^{r}(c, C(K))$ is not a lattice but it satisfies the countable interpolation property, by Theorem 3.1 of [17], which we have referred to in the introduction. If $K=\beta(\mathbb{N}) \backslash \mathbb{N}$, where $\beta(\mathbb{N})$ is the compactification of $\mathbb{N}$, then the space $F=C(K)$ has the countable interpolation property but it is not $\sigma$-Dedekind complete; see [15. 
Example 12. Suppose that $E=\mathcal{L}^{r}(c, C(K))$, where $K=\beta(\mathbb{N}) \backslash \mathbb{N}$. Then there exists $T \in E$, so that the supremum of $\{T, 0\}$ does not exist in $E$. If $T=T_{1}-T_{2}$, where $T_{1}, T_{2} \in E_{+}$and $L=T_{1}+T_{2}$ and $I=I_{L}$ is the order convex subspace of $E$ generated by $L$, the supremum $\{T, 0\}$ in $I$ does not exist. Indeed if we suppose that $G$ is the supremum of $\{T, 0\}$ in $I$, then this is the supremum of $\{T, 0\}$ in $E$, because for any $T^{\prime} \in E, T^{\prime} \geq T, 0$, we have that $T^{\prime}, L \geq T, 0$ and by the countable interpolation property there exists $G^{\prime} \in E$, so that

$$
T^{\prime}, L \geq G^{\prime} \geq T, 0 \text {. }
$$

By the definition of $I$ we have that $G^{\prime} \in I$; hence $T^{\prime} \geq G^{\prime} \geq G$. So we have that $G$ is also the supremum of $\{T, 0\}$ in $E$, a contradiction.

A sequence $\left\{x_{n}\right\}$ of an ordered Banach space $E$ is a positive basis of $E$ if $\left\{x_{n}\right\}$ is a Schauder basis of $E$ and $E_{+}=\left\{\sum_{n=1}^{\infty} a_{n} x_{n} \mid a_{n} \geq 0\right\}$. Note that by [16], Theorem 16.3, page 473 , if $E$ is an ordered Banach space with a positive basis $\left\{x_{n}\right\}$, then $\left\{x_{n}\right\}$ is unconditional (or equivalently $E$ is a Banach lattice with respect to an equivalent norm) if and only if $E_{+}$is generating and normal.

Theorem 13. Let $E$ be an ordered Banach space with a positive basis $\left\{x_{n}\right\}$ and generating positive cone $E_{+}$. If $F$ is an ordered Banach space with closed and normal positive cone $F_{+}$and if $F$ has the countable interpolation property, then $\mathcal{L}^{r}(E, F)$ has the countable interpolation property.

Proof. Suppose that $\left\{V_{p}\right\},\left\{W_{q}\right\}$ are sequences of $\mathcal{L}^{r}(E, F)$ such that $V_{p} \leq W_{q}$ for each $p, q \in \mathbb{N}$. Then $V_{p}\left(x_{n}\right) \leq W_{q}\left(x_{n}\right)$ for each $p, q \in \mathbb{N}$ and by the countable interpolation property of $F$, a sequence $\left\{y_{n}\right\}$ of $F$ exists so that

$$
V_{p}\left(x_{n}\right) \leq y_{n} \leq W_{q}\left(x_{n}\right) \text { for each } p, q \text { and each } n \text {. }
$$

For any $x=\sum_{n=1}^{\infty} a_{n} x_{n} \in E_{+}$, the sequence $\left\{a_{n}\right\}$ is positive and we have

$$
u=V_{1}\left(\sum_{i=m}^{n} a_{i} x_{i}\right) \leq v=\sum_{i=m}^{n} a_{i} y_{i} \leq w=W_{1}\left(\sum_{i=m}^{n} a_{i} x_{i}\right) .
$$

If $c$ is a constant of the normal cone $F_{+}$we have $0 \leq v-u \leq w-u$; therefore $\|v-u\| \leq c\|w-u\|$ from where we get that $\|v\| \leq 2(c+1) \max \{\|w\|,\|u\|\}$. Therefore we have

$$
\left\|\sum_{i=m}^{n} a_{i} y_{i}\right\| \leq 2(c+1) \max \left\{\left\|V_{1}\right\|,\left\|W_{1}\right\|\right\}\left\|\sum_{i=m}^{n} a_{i} x_{i}\right\|
$$

and we have that $\sum_{n=1}^{\infty} a_{n} y_{n}$ exists in $F$. For any $x=\sum_{n=1}^{\infty} a_{n} x_{n} \in E_{+}$we put

$$
T(x)=\sum_{n=1}^{\infty} a_{n} y_{n} .
$$

Then $T$ is positive homogeneous and additive in $E_{+}$and we extend $T$ in $E$ by the formula $T(x)=T\left(x_{1}\right)-T\left(x_{2}\right)$, where $x=x_{1}-x_{2}, x_{1}, x_{2} \in E_{+}$. Note that $T$ is well defined because if $x=y_{1}-y_{2}, y_{1}, y_{2} \in E_{+}$, we have $T\left(x_{1}\right)-T\left(x_{2}\right)=T\left(y_{1}\right)-T\left(y_{2}\right)$. By (18), we have that

$$
V_{p} \leq T \leq W_{q}
$$

for any $p, q$. Since $W_{q}-T \geq 0$, by the Lozanovsky theorem, $W_{q}-T$ is continuous and therefore regular. So $T$ is regular and $\mathcal{L}^{r}(E, F)$ has the countable interpolation property. 


\section{REFERENCES}

1. Y.A Abramovich and A.W Wickstead, The regularity of order bounded operators into $C(K)$ II, Quart. J. Math. Oxford 44 (1993), 257-270. MR1240470 (94h:47067)

2. A.A. Albanese, J. Bonet, and W.J. Ricker, Grothendieck spaces with the Dunford-Pettis property, Positivity 14 (2010), no. 1, 145-164. MR2596470 (2011b:46006)

3. C. D. Aliprantis and O. Burkinshaw, Positive Operators, vol. 119, Springer, Pure and Applied Math. Series, Academic Press, New York, 2006. MR2262133

4. C.D. Aliprantis and R. Tourky, Cones and Duality, vol. 84, Graduate Studies in Mathematics, Amer. Math. Soc., 2007. MR2317344 (2008k:46012)

5. O. Burkinshaw, Weak compactness in the order dual of a vector lattice, Transactions of the AMS 187 (1974), 183-201. MR0394098 (52:14904)

6. N. Danet, The Riesz decomposition property for the space of regular operators, Proceedings of the AMS 129 (2001), 539-542. MR.1707144 (2001e:47065)

7. P. G. Dodds, Sequential convergence in the order duals of certain classes of Riesz spaces, Transactions of the AMS 203 (1975). MR0358282 (50:10748)

8. A. Grothendieck, Sur les applications linéaires faiblement compactes d'espaces du type $C(K)$, Canada. J. Math. 5 (1953), 129-173. MR0058866 (15:438b)

9. C.B. Huijsmans and B. de Pagter, On z-ideals and d-ideals in Riesz spaces II, Indag. Math. 42 (1980), 391-408. MR.597997 (83c:46004a)

10. D. Ji, M. Craddock, and Q. Bu, Reflexivity and the Grothendieck property for positive tensor products in Banach lattices, Positivity 14 (2010), no. 1, 59-68. MR2596463 (2011b:46037)

11. H. E. Lacey, The Isometric Theory of Classical Banach Spaces, Springer-Verlag, New York, 1974. MR0493279 (58:12308)

12. H. P. Lotz, Weak* convergence in the dual of weak $L^{p}$, Israel Journal of Mathematics 176 (2010), 209-220. MR2653192 (2011j:46042)

13. P. Meyer-Nieberg, Banach Lattices, Springer-Verlag, 1991. MR1128093 (93f:46025)

14. I.A. Polyrakis and F. Xanthos, Cone characterization of Grothendieck spaces and Banach spaces containing $c_{0}$, Positivity, Vol. 15, No. 4 (2011), 677-693. MR2861605

15. G. L. Seever, Measures on F-spaces, Transactions of the AMS 133 (1968), 267-280. MR0226386 (37:1976)

16. I. Singer, Bases in Banach spaces - I, Springer-Verlag, Heidelberg and New York, 1970. MR0298399(45:7451)

17. A.W Wickstead, Spaces of operators with the Riesz separation property, Indag. Math. 6 (1995), 235-245. MR1338329 (96g:47032)

Department of Mathematics, National Technical University of Athens, Zographou 157 80, Athens, Greece

E-mail address: ypoly@math.ntua.gr

Department of Mathematics, National Technical University of Athens, Zographou 157 80, Athens, Greece

E-mail address: fxanthos@math.ntua.gr 